\title{
A black hole fundamental plane
}

\author{
Philip F. Hopkins \\ Harvard-Smithsonian Center for Astrophysics, 60 Garden Street, Cambridge, MA 02138, USA \\ email: phopkins@cfa.harvard.edu
}

\begin{abstract}
We study observed correlations between supermassive black hole (BHs) and the properties of their host galaxies, and show that the observations define a $\mathrm{BH}$ "fundamental plane" (BHFP), of the form $M_{\mathrm{BH}} \propto \sigma^{3.0 \pm 0.3} R_{e}^{0.43 \pm 0.19}$ or $M_{\mathrm{BH}} \propto M_{*}^{0.54 \pm 0.17} \sigma^{2.2 \pm 0.5}$, analogous to the FP of elliptical galaxies. The BHFP is preferred over a simple relation between $M_{\mathrm{BH}}$ and any of $\sigma, M_{*}, M_{\mathrm{dyn}}$, or $R_{e}$ alone at $>3 \sigma(99.9 \%)$ significance. The existence of this BHFP has important implications for the formation of supermassive BHs and the masses of the very largest black holes, and immediately resolves several apparent conflicts between the BH masses expected and measured for outliers in both the $M_{\mathrm{BH}}-\sigma$ and $M_{\mathrm{BH}}-M_{*}$ relations.
\end{abstract}

Keywords. galaxies: bulges, galaxies: evolution, galaxies: nuclei, quasars: general

\section{Do Observations Favor a Multi-Variable Correlation Between Black Hole Mass and Host Properties?}

Discoveries of correlations between the masses of supermassive black holes (BHs) in the centers of nearby galaxies and the properties of their host spheroids (Kormendy \& Richstone 1995) demonstrate a fundamental link between the growth of BHs and galaxy formation. A large number of similar correlations have now been identified, linking BH mass to host luminosity (Kormendy \& Richstone 1995), mass (Magorrian et al. 1998), velocity dispersion (Ferrarese \& Merritt 2000; Gebhardt et al. 2000), concentration or Sersic index (Graham et al. 2001; Graham \& Driver 2006), and binding energy (Aller \& Richstone 2007), among others. However, because these properties of host spheroids are themselves correlated, it is not clear whether any are in some sense more basic.

In Hopkins et al. (2007a) we consider the sample of local BHs for which masses have been reliably determined via either kinematic or maser measurements. Specifically, we adopt the sample of 38 local systems for which values of $M_{\mathrm{BH}}, \sigma, R_{e}, M_{\mathrm{dyn}}$, and bulge luminosities are compiled in Marconi \& Hunt (2003) and Häring \& Rix (2004). We wish to determine whether or not a simple one-to-one correlation between e.g. $M_{\mathrm{BH}}$ and $\sigma$ is a sufficient description of the data, or if there is evidence for additional dependence on a second parameter such as $R_{e}$ or $M_{*}$. The most efficient way to determine such a dependence is by looking for correlations between the residuals of the various projections of such a potential BHFP relation.

Figure 1 plots the correlation between $\mathrm{BH}$ mass $M_{\mathrm{BH}}$ and host bulge effective radius $R_{e}$ or bulge stellar mass $M_{*}$, all at fixed $\sigma$, where e.g. the residual of $\mathrm{BH}$ mass at a given $\sigma$ is

$$
\Delta \log \left(M_{\mathrm{BH}} \mid \sigma\right) \equiv \log \left(M_{\mathrm{BH}}\right)-\left\langle\log \left(M_{\mathrm{BH}}\right)\right\rangle(\sigma) .
$$

The figure demonstrates that there is a highly significant correlation between $M_{\mathrm{BH}}$ and $R_{e}$ or $M_{*}$ at fixed $\sigma$. We repeat this exercise in the figure, and demonstrate similarly that there is a highly significant correlation between $M_{\mathrm{BH}}$ and $\sigma$ or $M_{*}$ at fixed $R_{e}$, and between $M_{\mathrm{BH}}$ and $\sigma$ or $R_{e}$ at fixed $M_{*}$. This indicates that a simple one-variable correlation (e.g. a $M_{\mathrm{BH}}(\sigma)$, or $M_{\mathrm{BH}}\left(M_{*}\right), M_{\mathrm{BH}}\left(R_{e}\right)$ relation) is an incomplete description 

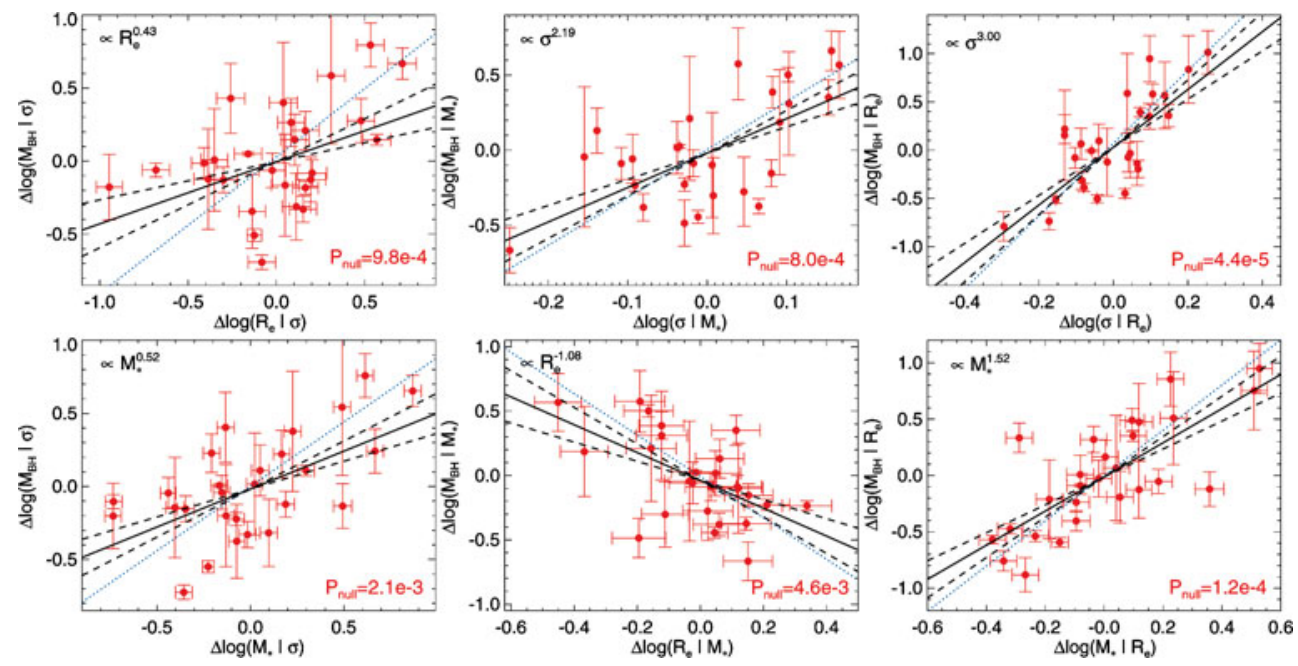

Figure 1. Left: Observed residual in BH mass as a function of host galaxy effective radius $R_{e}(t o p)$ or stellar mass $M_{*}$ (bottom), at fixed velocity dispersion $\sigma$ (equivalently, correlation between the residuals in the $M_{\mathrm{BH}}-\sigma$ and $R_{e}-\sigma$ or $M_{*}-\sigma$ relations at each $\sigma$ ). The fit to this residual correlation is shown with the black lines $( \pm 1 \sigma$ range in the best-fit correlation shown as dashed lines - note that they are strongly inconsistent with zero correlation), with the slope shown (dotted blue line shows the least-squares bisector). The probability of the null hypothesis of no correlation in the residuals (i.e. no systematic dependence of $M_{\mathrm{BH}}$ on $R_{e}$ or $M_{*}$ at fixed $\sigma)$ for the observed systems is shown (red $P_{\text {null }}$ ). Center: Residual in $M_{\mathrm{BH}}$ as a function of $\sigma$ or $R_{e}$ at fixed stellar mass $M_{*}$. Right: Residual in $M_{\mathrm{BH}}$ as a function of $\sigma$ or $M_{*}$ at fixed effective radius $R_{e}$. The observations imply a secondary "fundamental plane"-type correlation at $3 \sigma$ with respect to each of these variables.

of the observations. We therefore introduce a FP-like relation of the form

$$
M_{\mathrm{BH}} \propto \sigma^{\alpha} R_{e}^{\beta},
$$

which can account for these dependencies. Formally, we determine the combination of $(\alpha, \beta)$ which simultaneously minimizes the $\chi^{2} / \nu$ of the fit and the significance of the correlations between the residuals in $\sigma$ and $M_{\mathrm{BH}}$ (or $R_{e}$ and $M_{\mathrm{BH}}$ ). This yields a best-fit BHFP relation

$$
\begin{aligned}
\log \left(M_{\mathrm{BH}}\right)= & 8.33+3.00( \pm 0.30) \log \left(\sigma / 200 \mathrm{~km} \mathrm{~s}^{-1}\right) \\
& +0.43( \pm 0.19) \log \left(R_{e} / 5 \mathrm{kpc}\right) .
\end{aligned}
$$

As expected, the residuals of $M_{\mathrm{BH}}$ with respect to these fundamental plane relations, at fixed $R_{e}$ and fixed $\sigma$, show no systematic trends and are consistent with small intrinsic scatter. The introduction of a BHFP eliminates the strong systematic correlations between the residuals, yielding flat errors as a function of $\sigma$ and $R_{e}$.

Figure 1 demonstrates the significance with which the observations rule out both a pure BH-host mass relation (either $M_{\mathrm{BH}} \propto M_{*}$ or $M_{\mathrm{BH}} \propto M_{\mathrm{dyn}}$ ) and a pure $M_{\mathrm{BH}}-\sigma$ relation. However, when fitting to a form $M_{\mathrm{BH}} \propto \sigma^{\alpha} R_{e}^{\beta}$, there is still some degeneracy between the slopes $\alpha$ and $\beta$ (roughly along the axis $\beta \approx(4-\alpha) / 2$ ). Figure 2 illustrates the degree of this degeneracy and the extent to which, for example, a BHFP with $M_{\mathrm{BH}} \propto \sigma^{3} R_{e}^{1 / 2}$ is favored over a pure $M_{\mathrm{BH}}-M_{\mathrm{dyn}}$ relation. We plot the likelihood of a residual correlation between $M_{\mathrm{BH}}$ and $R_{e}$ or $\sigma$ at fixed $\sigma^{\alpha} R_{e}^{\beta}$, as a function of the slope $\alpha$ (marginalizing over $\beta$ and other fit parameters at each $\alpha$, although $\beta(\alpha)$ roughly follows the axis of 

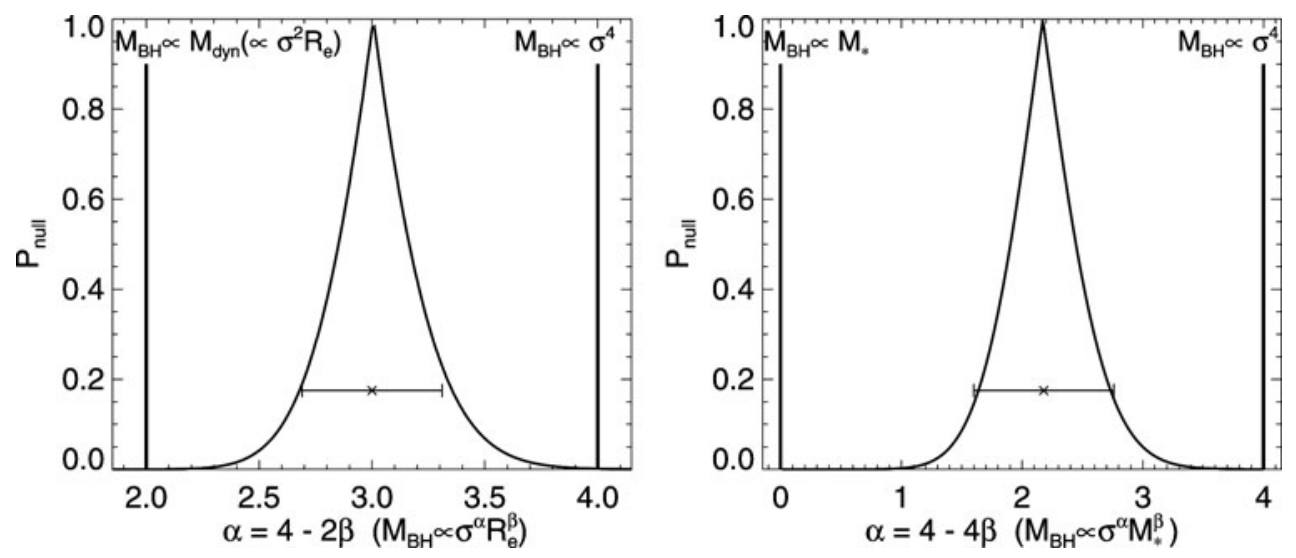

Figure 2. Probability that there is no remaining trend or correlation among the residuals of the $M_{\mathrm{BH}}$-host relation ( $P_{\mathrm{null}}$, as in Figure 1 ), as a function of the slope $\alpha$ for a correlation of the form $M_{\mathrm{BH}} \propto \sigma^{\alpha} R_{e}^{\beta}$ (top) or $M_{\mathrm{BH}} \propto \sigma^{\alpha} M_{*}^{\beta}$ (bottom). For each value of $\alpha$, we marginalize over $\beta$ and the correlation normalization to find the best fit, but there is a rough degeneracy between the best fit $\alpha$ and $\beta\left(\beta \approx(4-\alpha) / 2\right.$ for $M_{\mathrm{BH}} \propto \sigma^{\alpha} R_{e}^{\beta}, \beta \approx(4-\alpha) / 4$ for $\left.M_{\mathrm{BH}} \propto \sigma^{\alpha} M_{*}^{\beta}\right)$. Lines show $P_{\text {null }}$ for the observations, points show the best fit (in a $\chi^{2}$ sense) BHFP relation and errors. Thick black lines show the $\alpha$ corresponding to a pure $M_{\mathrm{BH}}-\sigma, M_{\mathrm{BH}}-M_{\mathrm{dyn}}$, or $M_{\mathrm{BH}}-M_{*}$ relation, all of which are ruled out at similar $(\sim 3 \sigma)$ significance.

degeneracy above). We then repeat this exercise for the alternate representation of the fundamental plane, $M_{\mathrm{BH}} \propto \sigma^{\alpha} M_{*}^{\beta}$.

There are possible correlations which cannot be clearly discriminated by the present data. Both a pure relation between $\mathrm{BH}$ mass and spheroid binding energy, of the form $M_{\mathrm{BH}} \propto\left(M_{*} \sigma^{2}\right)^{2 / 3}$, as studied in Aller \& Richstone (2007), for example, and a mixed relation of the form $M_{\mathrm{BH}} \propto M_{*}^{1 / 2} \sigma^{2}$ presented in Hopkins et al. (2007b), are within the $\sim 1 \sigma$ allowed range of the data. It is worth noting that simply expanding the number of observed sources will not necessarily break these degeneracies. Rather, to increase the constraining power of the observations, a larger baseline is needed, including (in particular) a larger sample of objects which lie off the mean $R_{e}-\sigma$ or $M_{*}-\sigma$ relations (and thus extend the baseline in the residual-residual space which properly constrains the FP slopes). Finally, we noted above that Figure 1 is essentially unchanged if we consider residuals with respect to just linear (i.e. pure power law) $M_{\mathrm{BH}}-\sigma$-type relations, as a consequence of there being no significant evidence in our data for a log-quadratic or higher-order correlation. Allowing log-quadratic terms in our fundamental plane fits, we find a best fit to the observations of the form

$$
\begin{aligned}
\log \left(M_{\mathrm{BH}}\right)=8.06 & +(2.8 \pm 0.4) \tilde{\sigma}+(0.48 \pm 0.18) \tilde{r} \\
& -(2.1 \pm 2.3) \tilde{\sigma}^{2}+(0.31 \pm 0.25) \tilde{r}^{2}
\end{aligned}
$$

(where $\tilde{\sigma} \equiv \log \left[\sigma / 200 \mathrm{~km} \mathrm{~s}^{-1}\right]$ and $\tilde{r} \equiv \log \left[R_{e} / 3 \mathrm{kpc}\right]$ ). The linear BHFP coefficients in $\sigma$ and $R_{e}$ are similar to those we found before, and their significance is not much changed - this illustrates that the FP behavior we find cannot simply trade off with or be equally well-represented by a log-quadratic dependence (i.e. one cannot eliminate the residual dependence of $M_{\mathrm{BH}}$ on $R_{e}$ at fixed $\sigma$ by adding a log-quadratic or higher-order term in $\sigma$ ). The log-quadratic terms are at most significant at the $\sim 1 \sigma$ level. This is also true if we add just one of the two log-quadratic terms - adding a log-quadratic term in just $R_{e}$ yields a coefficient $(0.16 \pm 0.25) \tilde{r}^{2}$, and adding one in just $\sigma$ gives a coefficient 
$(-0.28 \pm 2.15) \tilde{\sigma}^{2}$. This is similar to the finding of Wyithe $(2006)$, who estimates $<1 \sigma$ significance for the addition of log-quadratic terms in any of $\sigma, M_{\mathrm{dyn}}$, or $M_{*}$.

\section{Conclusions}

We study the correlation between observed central BH mass and host galaxy properties, and find that the systems lie on a BH "fundamental plane" (BHFP), of the form $M_{\mathrm{BH}} \propto$ $\sigma^{3.0} R_{e}^{0.5}$ or $M_{\mathrm{BH}} \propto M_{*}^{0.5-0.7} \sigma^{1.5-2.0}$, analogous to the FP of spheroids. Specifically, there are significant (at $>99.9 \%$ confidence) trends in the residuals of the $M_{\mathrm{BH}}-\sigma$ relation with $M_{*}$ and $R_{e}$ at fixed $\sigma$, and likewise in the $M_{\mathrm{BH}}-M_{*}$ relation (with $\sigma$ or $R_{e}$ at fixed $M_{*}$ ). This provides a new paradigm for understanding the traditional relations between $\mathrm{BH}$ mass and either bulge velocity dispersion or mass. These correlations (as well as those with other bulge properties such as effective radius, central potential, dynamical mass, concentration, Sersic index, and bulge binding energy) are all projections of the same fundamental plane relation. Just as the Faber-Jackson relation between e.g. stellar mass or luminosity and velocity dispersion $\left(M_{*}-\sigma\right)$ is understood as a projection of the more fundamental relation between $M_{*}, \sigma$, and $R_{e}$, so too is the $M_{\mathrm{BH}}-\sigma$ relation $\left(M_{\mathrm{BH}} \propto \sigma^{4}\right)$ a projection of the more fundamental relation $M_{\mathrm{BH}} \propto \sigma^{3} R_{e}^{0.5}$. Recognizing this resolves the nature of several apparent outliers in the $M_{\mathrm{BH}}-\sigma$ relation, which simply have unusual $\sigma$ values for their stellar masses or effective radii, and eliminates the strong correlations between residuals. Improved measurements of the host properties of systems with well-measured BHs can significantly improve constraints on the BHFP. The present observations demand a correlation of the form $M_{\mathrm{BH}} \propto \sigma^{\alpha} M_{*}^{\beta}$ over a simple correlation with either $\sigma$ or $M_{*}$ at $\gtrsim 3 \sigma$ confidence. Already, this puts strong constraints on theoretical models of $\mathrm{BH}$ growth and evolution - $\mathrm{BH}$ mass does not simply scale with the star formation (stellar mass) or virial velocity of the host galaxy. However, there is still a substantial degeneracy between the slopes $\alpha$ and $\beta$ (roughly along the axis $\beta \approx 1-\alpha / 4$ ). For example, the existing data do not allow us to significantly distinguish a pure correlation with spheroid binding energy $M_{\mathrm{BH}} \propto\left(M_{*} \sigma^{2}\right)^{2 / 3}$, as detailed in Aller \& Richstone (2007) from the marginally favored relation $\propto M_{*}^{1 / 2} \sigma^{2}$. Both suggest that the ability of BHs to self-regulate their growth must be sensitive to the potential well at the center of the galaxy (and therefore to galactic structure), but the difference could reveal variations in the means by which $\mathrm{BH}$ feedback couples to the gas on these scales.

\section{References}

Aller, M. C. \& Richstone, D. O. 2007, ApJ, accepted, arXiv:0705.1165v1 [astro-ph]

Ferrarese, L. \& Merritt, D. 2000, ApJ, 539, L9

Gebhardt, K., et al. 2000, ApJ, 539, L13

Graham, A. W. \& Driver, S. P. 2006, ApJ, in press [astro-ph/0607378]

Graham, A. W., Erwin, P., Caon, N., \& Trujillo, I. 2001, ApJ, 563, L11

Häring, N. \& Rix, H.-W. 2004, ApJ, 604, L89

Hopkins, P. F., Hernquist, L., Cox, T. J., Robertson, B., \& Krause, E. 2007a, ApJ, accepted, arXiv:0707.4005v1 [astro-ph]

Hopkins, P. F., Hernquist, L., Cox, T. J., Robertson, B., \& Krause, E. 2007b, ApJ, accepted [astro-ph/0701351]

Kormendy, J. \& Richstone, D. 1995, ARAESA, 33, 581

Magorrian, J., et al. 1998, AJ, 115, 2285

Marconi, A. \& Hunt, L. K. 2003, ApJLetters, 589, L21

Tremaine, S., et al. 2002, ApJ, 574, 740

Wyithe, J. S. B. 2006, MNRAS, 365, 1082 